\title{
RESPOSTA ESPECTRAL DE SOLOS EM RAZÃO DO ÂNGULO DE VISADA, DA UMIDADE E DA RUGOSIDADE SUPERFICIAL ${ }^{1}$
}

\author{
LUCIANO JOSÉ DE OLIVEIRA ACCIOLY² e ALFREDO RAMON HUETE ${ }^{3}$
}

\begin{abstract}
RESUMO - Este trabalho teve por objetivo avaliar as variações do fator de refletância bidirecional (FRB) de três séries de solo (McAllister, Stronghold e Epitaph) da microbacia experimental de Walnut Gulch (Arizona, EUA) em razão do ângulo de visada, da rugosidade superficial e do teor de umidade. Foram consideradas as faixas espectrais do visível e do infravermelho próximo e médio presentes no sensor TM, e os resultados foram expressos em termos de FRB em relação à resposta no Nadir (FRB relativo). O anisotropismo variou de solo para solo e foi maior nas menores faixas espectrais, nos ângulos de visada maiores localizados na direção do retroespalhamento, nos ângulos solar-zenitais maiores, e na condição de solo seco. No solo Epitaph (único solo submetido ao estudo de rugosidade) o anisotropismo foi também maior na superfície mais rugosa. Entretanto, uma melhor diferenciação entre as superfícies lisa e rugosa do solo Epitaph foi obtida na direção do espalhamento da energia refletida. Diferenças na escala e nos métodos de obtenção dos dados são apontadas como causas do realce do comportamento anisotrópico dos dados obtidos em condições de laboratório, em comparação com os dados de campo.
\end{abstract}

Termos para indexação: sensoriamento remoto, refletância, espectrometria.

\section{SOIL SPECTRAL RESPONSE IN RELATION TO VIEWING ANGLE, SOIL MOISTURE AND SURFACE ROUGHNESS}

\begin{abstract}
The objective of this study was to characterize the bi-directional reflectance factor (BRF) of three soil series (McAllister, Stronghold, and Epitaph) located at the Walnut Gulch Experimental Watershed (Arizona, USA) as a function of the viewing angle, soil moisture and surface roughness. Soil spectra were taken in the visible, near and mid-infrared regions, convolved to match the Landsat-TM bands, and the results were normalized to the Nadir response and expressed as relative BRF. The anisotropic behavior varies from soil to soil and it was higher when the following conditions were taken combined or individually: shorter wavelengths, higher viewing and solar zenith angles, in the backscattering direction, soil in the dry condition as opposed to wet condition, and in the rough surface as compared to the smooth surface of Epitaph soil series (the only soil tested for the effect of roughness). Rough and smooth surfaces of Epitaph soil, however, were better discriminated in the forward scattering direction. Differences in scale and methods used to obtain the spectral curves were pointed out as responsible for the enhancement of the anisotropic behavior of the soils for lab results as compared to field results.
\end{abstract}

Index terms: remote sensing, reflectance, spectrometry.

\footnotetext{
${ }^{1}$ Aceito para publicação em 2 de março de 2000.

${ }^{2}$ Eng. Agrôn., Ph.D., Embrapa-Centro Nacional de Pesquisa de Solos (CNPS), Unidade de Execução de Pesquisa e Desenvolvimento (UEP-Recife), Rua Antonio Falcão, 402, CEP 51020-240 Recife, PE. E-mail: oaccioly@elogica.com.br

${ }^{3}$ Eng. Agrôn., Ph.D., Soil Water and Environmental Science Department, The University of Arizona, 85721, Tucson, AZ. E-mail: ahuete@ag.arizona.edu
}

\section{INTRODUÇÃO}

As variações no comportamento espectral dos solos levando-se em consideração as mudanças na geometria que combinam os ângulos de visada e de incidência da fonte de iluminação têm sido estudadas por vários pesquisadores (Coulson et al., 1965; 
Coulson, 1966; Eaton \& Dirmhirn, 1979; Irons et al., 1989). Tanto em dados de laboratório (Coulson et al., 1965; Coulson, 1966) quanto nos dados coletados sob condição de campo (Coulson \& Reynolds, 1971; Eaton \& Dirmhirn, 1979; Kimes, 1983; Kimes \& Sellers, 1985; Kimes et al., 1985), a maioria dos solos apresenta um comportamento anisotrópico que é assimétrico em relação à linha do Nadir. O conhecimento desse comportamento (também conhecido como fator de refletância bidirecional ou FRB) é indispensável para a extração de informações sobre os solos de uma dada região a partir de imagens de satélite, principalmente dos sensores que apresentam grande visão angular (e.g. NOAA-AVHRR).

Variações no FRB dos solos dependem de vários fatores, tais como: a textura, a constituição das partículas do solo, a rugosidade superficial, o teor de umidade, o encrostamento. Na maioria dos solos, o FRB aumenta quando o ângulo de visada aumenta em relação ao Nadir na direção do retroespalhamento. As areias, entretanto, podem apresentar um comportamento anisotrópico em que o FRB aumenta na direção do espalhamento da energia refletida (Coulson, 1966). O pronunciado pico de refletância na direção do retroespalhamento tem sido atribuído à redução do efeito da sombra em superfícies com elevado grau de rugosidade (Eaton \& Dirmhirn, 1979). Uma função empírica sugerida por Minnaert (Irons \& Smith, 1990) vem sendo utilizada para analisar os efeitos da topografia sobre dados de sensores remotos. Por meio da constante geométrica $\mathrm{k}$ da função de Minnaert, é possível projetar o FRB de determinada superfície. Ao estudar as alterações no valor dessa constante, Irons \& Smith (1990) notaram que valores de k dependiam não só da rugosidade superficial mas também da forma e orientação dos agregados superficiais. Jackson et al. (1990) estudaram o efeito das variações nos ângulos de elevação solar e de visada sobre o FRB de diferentes superfícies niveladas, gradeadas com a grade de discos e sulcadas com uma grade sulcadora. Esses autores determinaram que em qualquer condição de rugosidade, a radiação refletida foi maior na direção do retroespalhamento, com pico para um ângulo de visada de $44^{\circ}$. A superfície mais rugosa (sulcada) foi a que apresentou maior grau de anisotropismo.
Em determinado solo, as curvas características do FRB são diferentes quando são consideradas amostras úmidas e secas. Quando se adiciona água a amostras de solo, o efeito é de redução generalizada da radiação refletida no intervalo espectral que vai de 0,4 a 2,6 $\mu \mathrm{m}$ (Bowers \& Hanks, 1965; Hoffer \& Johannsen, 1969; Curran et al., 1990; Epiphânio et al., 1992). Embora alguns autores (Curran et al., 1990) mencionem que a redução na radiação refletida pode alcançar um fator de 2 a 3, o formato da curva espectral de determinado solo não é substancialmente alterado pela adição de água. O efeito de redução na radiação refletida após a adição de água a amostras de solos tem-se mostrado dependente da cor do solo (Curran et al., 1990; Epiphânio et al., 1992). Em um estudo conduzido em 111 amostras de solos tropicais, Epiphânio et al. (1992) encontraram que Latossolos de coloração vermelho-escuro apresentaram relativamente menor redução na radiação refletida do que Latossolos de cor vermelho mais claro após o seu umedecimento.

Fatores tais como a geometria, que combinam os ângulos de visada e de incidência da fonte de iluminação, a rugosidade superficial e a condição de umidade (seco x úmido) afetam a resposta espectral de determinado solo.

Esta pesquisa teve como propósito a caracterização do FRB dos solos da microbacia experimental de Walnut Gulch, em razão do ângulo de visada, da condição de umidade e da rugosidade superficial.

\section{MATERIAL E MÉTODOS}

As medições de FRB foram feitas em três séries de solos presentes na microbacia experimental de Walnut Gulch no sudeste do Estado do Arizona $\left(31,7^{\circ} \mathrm{N}\right.$ e $\left.110,0^{\circ} \mathrm{W}\right)$, EUA. As séries de solo e as respectivas classificações pela Soil Taxonomy (Estados Unidos, 1975) na qualidade de famílias foram as seguintes: Stronghold (Coarse-loamy, mixed, thermic Ustollic Calciargids), McAllister (Fineloamy, mixed, thermic Ustollic Calciargids) e Epitaph (Fine, montmorillonitic, thermic Leptic Haplotorrets). Na classificação brasileira (Embrapa, 1981) esses solos corresponderiam a: Podzólico Acinzentado Ta eutrófico, textura média com carbonato, fase caatinga hiperxerófila; Podzólico Vermelho-Amarelo Ta eutrófico, textura argilosa com carbonato, fase caatinga hiperxerófila; e Vertissolo, textura argilosa, para Stronghold, McAllister e 
Epitaph, respectivamente. O solo Epitaph é derivado de rochas vulcânicas de coloração escura, e ocupa parte das áreas planas da microbacia. McAllister e Stronghold estão entre os solos mais desenvolvidos da microbacia, mas apresentam coloração superficial contrastante. Enquanto McAllister tem coloração avermelhada, Stronghold apresenta-se superficialmente como um solo cinza-claro na superfície. Algumas das características físicas e químicas desses solos são apresentadas na Tabela 1 .

No campo, o FRB foi medido na estação seca de 1995/96, durante os meses de maio e junho. As áreas amostradas foram escolhidas em solo exposto com, no máximo, $5 \%$ de cobertura vegetal verde. Nessas medições foi utilizado um espectrorradiômetro ("Spectron" modelo SE590), com cobertura espectral que vai de 0,45 a $0,90 \mu \mathrm{m}$ e $0,01 \mu \mathrm{m}$ de intervalo amostral e $15^{\circ} \mathrm{de}$ imageamento. $\mathrm{O}$ espectrorradiômetro foi montado a 2,3 m de altura no aparelho de FRB descrito por Jackson et al. (1990). Este aparelho permite o ajuste manual do ângulo de visada de $-45^{\circ}$ (na direção do retroespalhamento) a $45^{\circ}$ (direção do espalhamento), com incrementos de $5^{\circ}$. As medições foram realizadas no intervalo de ângulos e seguindo o incremento permitido pelo aparelho de FRB, no plano principal e em um ângulo solar-zenital de $32 \pm 2^{\circ}$ e em condições de céu claro. Para avaliação do efeito da rugosidade na resposta espectral dos solos, foram ainda utilizados os seguintes ângulos solar-zenitais: $40 \pm 2^{\circ}$ e
$50 \pm 2^{\circ}$. Imediatamente após a medição do FRB em solo seco, a superfície do solo foi pulverizada com água para as leituras com solo úmido. Durante o umedecimento das superfícies dos solos, cuidou-se de evitar encharcamento e espelhos d'água, procurando-se manter constante a quantidade de água aplicada. Medições da resposta espectral de uma placa-padrão branca de "spectralon" calibrada foram realizadas imediatamente antes e imediatamente após as leituras sob condição de solo seco e umedecido. Um software especificamente desenvolvido para as condições locais de obtenção dos dados e que levava em consideração os coeficientes de calibração da placa-padrão, foi utilizado no tratamento dos dados, de saída do Spectron SE590. Os dados espectrorradiométricos foram também processados simulando as bandas $1(0,45$ a $0,52 \mu \mathrm{m})$, $2(0,52$ a $0,60 \mu \mathrm{m}), 3(0,63$ a $0,69 \mu \mathrm{m})$ e $4(0,76$ a $0,90 \mu \mathrm{m})$ do sensor TM do Landsat-5.

Amostras de solo foram retiradas dos locais onde foram efetuadas as medições de campo, peneiradas (peneira com $2 \mathrm{~mm}$ de malha) e encaminhadas ao Reflectance Laboratory (RELAB) na Universidade de Brown. As curvas espectrais do RELAB foram obtidas com o instrumento "Bidirectional Spectrometer" para a faixa espectral de 0,4 a $2,5 \mu \mathrm{m}$ com intervalo espectral de $0,005 \mu \mathrm{m}$. O espectrômetro do RELAB opera com lâmpada de halogênio e placa-padrão de "halon" prensado (Reflectance Experiment Laboratory, 1993). As medições

TABELA 1. Resultados das análises físicas e químicas das séries de solo McAllister, Stronghold e Epitaph.

\begin{tabular}{|c|c|c|c|}
\hline \multirow[t]{2}{*}{ Análise } & \multicolumn{3}{|c|}{ Série de solo } \\
\hline & McAllister & Stronghold & Epitaph \\
\hline \multicolumn{4}{|l|}{ Textura $^{1}$} \\
\hline Areia (g/kg) & 680 & 850 & 350 \\
\hline Silte $(\mathrm{g} / \mathrm{kg})$ & 200 & 120 & 390 \\
\hline $\operatorname{Argila~}(\mathrm{g} / \mathrm{kg})$ & 120 & 30 & 260 \\
\hline Carbono orgânico $(\mathrm{g} / \mathrm{kg})^{2}$ & 3,2 & 5,6 & 4,0 \\
\hline Ferro total $(\mathrm{g} / \mathrm{kg})^{2}$ & 16,1 & 11,1 & 19,7 \\
\hline \multicolumn{4}{|l|}{ Cor $^{3}$} \\
\hline \multicolumn{4}{|l|}{ Amostra seca } \\
\hline Matiz & 3,84 & 4,63 & 4,45 \\
\hline Valor & 4,41 & 4,91 & 2,79 \\
\hline Croma & 4,50 & 4,63 & 2,73 \\
\hline \multicolumn{4}{|l|}{ Amostra úmida } \\
\hline Matiz & 3,67 & 4,41 & 4,06 \\
\hline Valor & 3,42 & 3,53 & 2,98 \\
\hline Croma & 3,59 & 2,55 & 2,43 \\
\hline Rugosidade (comprimento relativo de arco) & 1,11 & 1,07 & 1,18 \\
\hline
\end{tabular}

\footnotetext{
1 Método do Densímetro (Embrapa, 1997).

2 Segundo metodologia descrita por Embrapa (1997).

3 Valores medidos com o Chromameter e transformados segundo Post et al. (1993).
} 
da resposta espectral do RELAB foram realizadas em relação aos ângulos de visada de $-35^{\circ}$ e $35^{\circ}$, ângulo de iluminação de $25^{\circ}$, e no plano principal.

Uma vez que só foi possível observar níveis de rugosidade superficiais contrastantes na série de solo Epitaph, esta unidade foi escolhida para avaliação dos efeitos da rugosidade superficial sobre suas curvas espectrais. As medições de rugosidade superficial foram realizadas em condições de campo, com o aparelho de avaliação da microrrugosidade (Simanton et al., 1971). Esse aparelho é composto por 100 pinos distribuídos em intervalos de $1 \mathrm{~cm}$, verticalmente orientados, que, quando desprendidos, geram o perfil da rugosidade superficial. O perfil foi delineado em papel milimetrado, e, posteriormente, digitalizado. O resultado foi expresso em termos de comprimento de arco em relação a uma superfície perfeitamente plana cujo comprimento relativo de arco é de $1 \mathrm{~m}$. Dez perfis de rugosidade no plano principal foram obtidos de cada tipo de rugosidade superficial (lisa, intermediária e rugosa) e de cada ângulo solar-zenital testado.

Os fragmentos de rocha presentes em cada tipo de rugosidade superficial foram avaliados com base nos critérios estabelecidos pelo Soil Survey Manual (Estados Unidos, 1993).

\section{RESULTADOS E DISCUSSÃO}

\section{Curvas espectrais obtidas no campo}

Os dados processados nas bandas do sensor TM Landsat-5 são apresentados na Fig. 1 nas condições de solo seco e umedecido. Esses dados foram normalizados para a resposta espectral do ângulo de visada do Nadir e expressos como FRB relativo. As três séries de solo apresentaram forte comportamento anisotrópico assimétrico em relação ao Nadir. O grau de anisotropismo diminuiu após o umedecimento da superfície dos solos, mostrando a dependência do FRB em relação à situação da umidade do solo. Após o umedecimento houve uma generalizada tendência ao achatamento das curvas do FRB relativo. Trabalhando com valores absolutos, Bowers \& Hanks (1965) encontraram redução nos valores de refletância, no visível e no infravermelho próximo, de um solo de textura franco-siltosa à medida que seu teor de umidade era aumentado. Em todas as bandas do TM e em ambas as condições (seco e umedecido), o FRB relativo aumentou à medida que se aumentou o ângulo de visada na direção do retroespalhamento até atingir um valor máximo (para aproxi- madamente $-35^{\circ}$ ), e diminuiu gradualmente à medida que o ângulo de visada aumentou no sentido do espalhamento da radiação refletida. Em decorrência da redução no ângulo solar-zenital, o valor de máximo FRB se deslocou de $-35^{\circ}$ para $-30^{\circ}$.

Maior anisotropismo foi apresentado pela série de solo Epitaph, seguido por McAllister e Stronghold (Fig. 1). Além de ser influenciado pela umidade, o comportamento anisotrópico de dois dos três solos foi também dependente da faixa espectral considerada. Desta forma, a resposta espectral na banda 1 (azul) apresentou maior grau de anisotropismo do que nas demais bandas.

A dependência do FRB em relação ao comprimento de onda considerado foi mais notada em relação à série de solo Epitaph. Neste solo e no ângulo de visada, em que o FRB relativo foi máximo (aproximadamente $-35^{\circ}$ ), a resposta espectral na banda azul foi aproximadamente $15 \%$ superior à encontrada na banda 4, localizada no infravermelho próximo. Em comparação com os resultados apresentados pelo solo Epitaph, a dependência do anisotropismo da resposta espectral em relação ao comprimento de onda foi mínima no solo Stronghold, e intermediária no solo McAllister, quando considerados os dados de campo do FRB. Essa menor dependência do anisotropismo do solo Stronghold, em relação ao comprimento de onda considerado, está associada ao baixo valor da rugosidade superficial apresentado por esse solo (Tabela 1).

Para uma análise mais pormenorizada do efeito do umedecimento dos solos sobre o FRB relativo, a Fig. 2 mostra a relação entre FRB relativo em condições de solo seco e em condições de solo úmido no ângulo de visada de $-30^{\circ}$ (nas proximidades do valor máximo de FRB), seu simétrico $30^{\circ}$ e Nadir ao longo da faixa do espectro considerada neste estudo. A relação FRB seco/FRB úmido foi maior no solo Epitaph do que nos outros dois solos, em qualquer dos três ângulos de visada considerados. Em todos os solos considerados, esta relação foi maior quando se considera a resposta espectral na direção do retroespalhamento $\left(-30^{\circ}\right)$, e diminuiu quando o ângulo de visada se deslocou na direção do espalhamento da energia refletida $\left(30^{\circ}\right)$. A relação FRB seco/FRB úmido mostrou-se dependente do comprimento de onda, notadamente no solo Epitaph. 

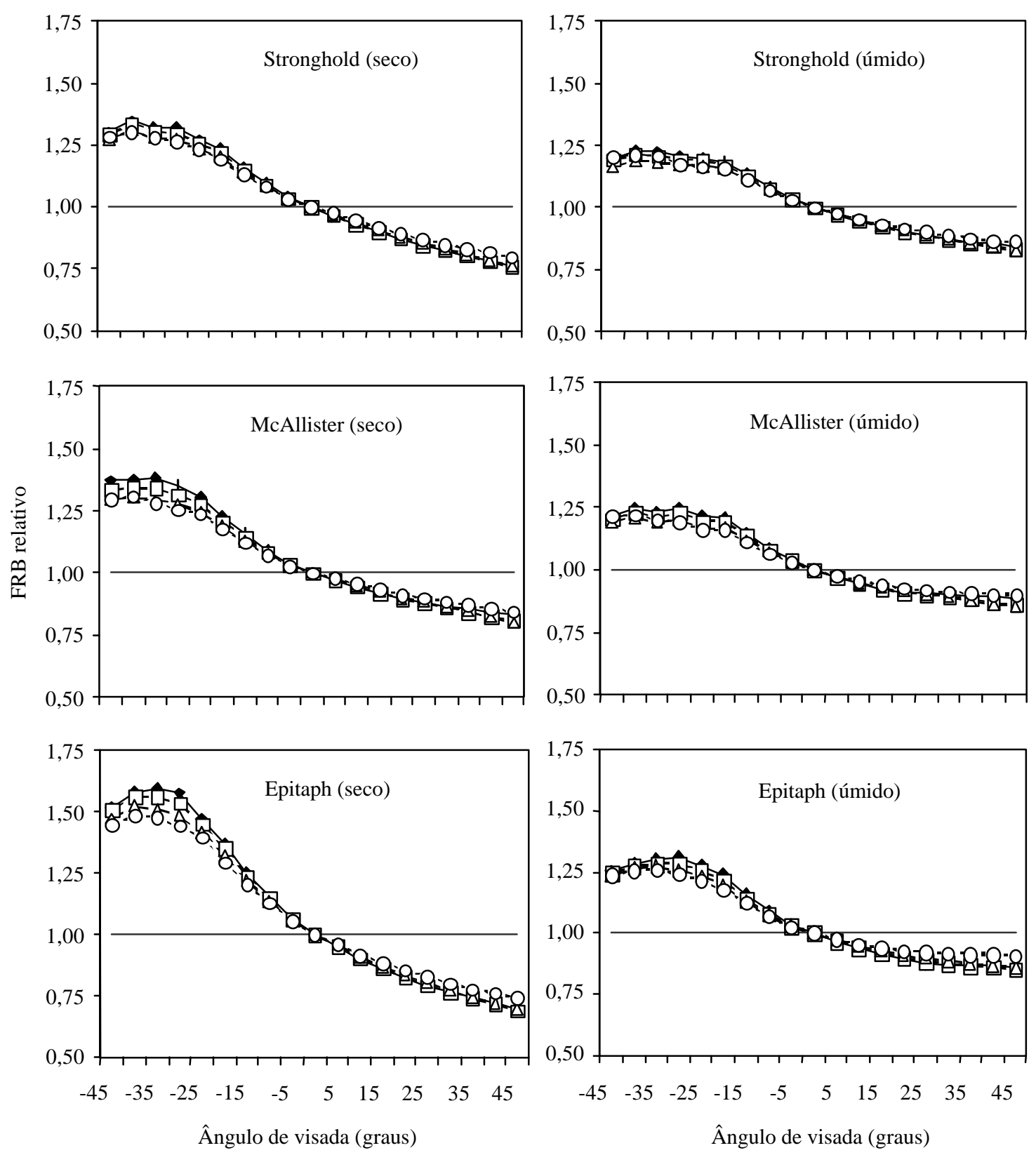

$\rightarrow \sim$ Banda $1 \quad-\square-$ Banda $2 \quad-$ Banda $3 \quad$ B $\quad-$ Banda 4

FIG. 1. Variação do fator de refletância bidirecional (FRB) obtido no campo nas condições de solo seco e úmido, em razão de diferentes ângulos de visada. (Os dados foram normalizados em relação ao Nadir; os símbolos indicam a resposta espectral das primeiras quatro bandas do sensor TM/Landsat 5 , simulados a partir dos dados espectrorradiométricos). 
Assim nessa série de solo, a relação ficou entre 1,8 e 1,9 no visível, e caiu para valores situados no intervalo de 1,6 e 1,7 no infravermelho próximo, quando
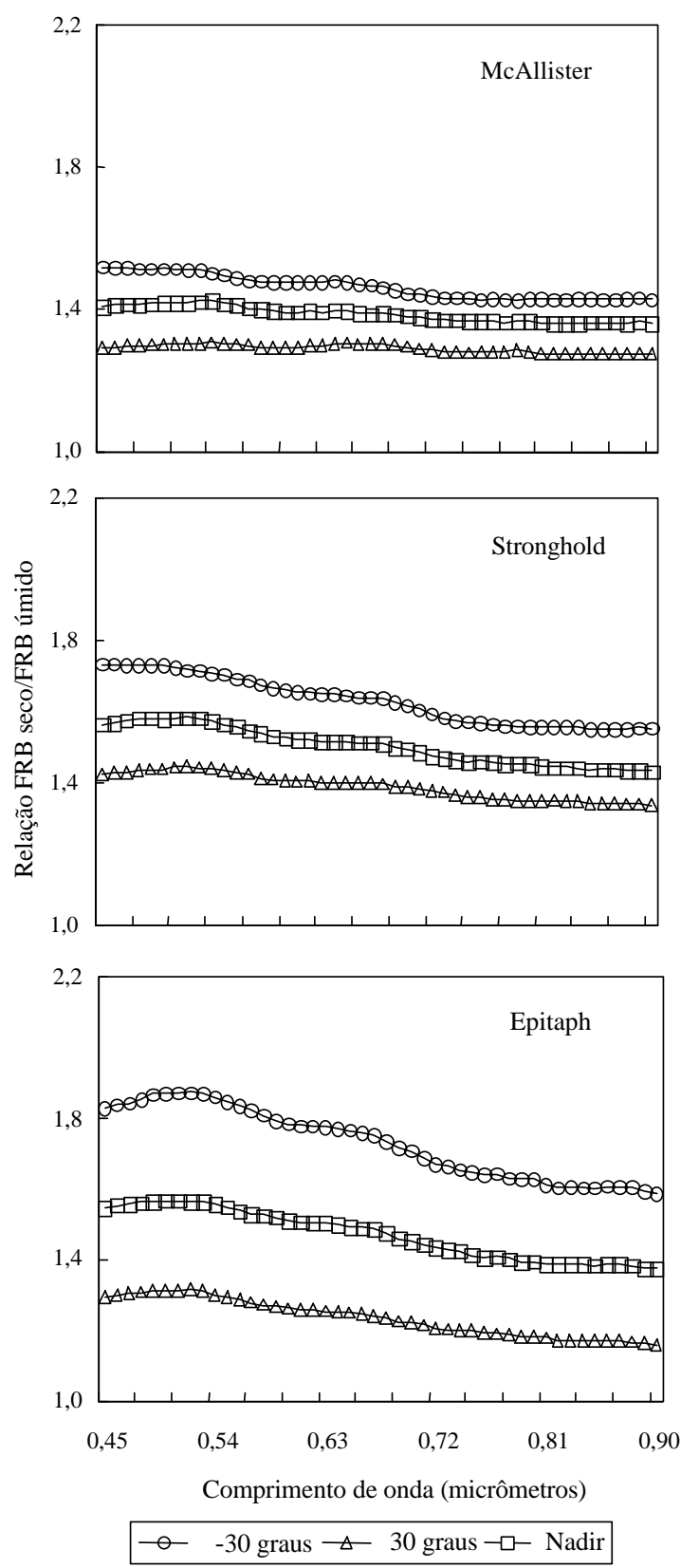

FIG. 2. Relação entre os valores de FRB relativo obtidos nas condições de solo seco e úmido, nos ângulos de visada do Nadir, -30 e 30 graus. considerados os valores de FRB obtidos no ângulo de visada de $-30^{\circ}$. As variações na relação FRB seco/ FRB úmido foram mínimas, quando considerados tanto o comprimento de onda quanto o ângulo de visada na série de solo McAllister, e intermediárias, na série de solo Stronghold. Tais resultados indicaram que os valores de FRB obtidos sob diferentes condições de umidade foram afetados por características intrínsecas do solo, pelo comprimento de onda e pela geometria determinada pelos ângulos de visada e da fonte de iluminação. Essas diferenças foram acentuadas no visível e quando o sensor esteve posicionado na direção do retroespalhamento. Nessa posição, o sinal recebido pelo sensor é menos influenciado pela sombra causada pela rugosidade superficial do solo (Coulson, 1966).

\section{Curvas espectrais obtidas no laboratório}

A Fig. 3 apresenta as curvas espectrais obtidas no RELAB referentes aos três ângulos de visada considerados: Nadir, $-35^{\circ}$ e $35^{\circ}$. Assim, como nos resultados obtidos no campo, os valores de FRB foram superiores na direção do retroespalhamento $\left(-35^{\circ}\right)$, seguidos daqueles que foram obtidos no Nadir e na direção do espalhamento da energia refletida $\left(35^{\circ}\right)$. Todas as curvas apresentaram um incremento monotônico nos valores de FRB a partir do azul, com valores máximos atingidos na região do infravermelho médio. Bandas de absorção estiveram presentes em todos os três solos estudados, porém em diferentes níveis de intensidade. Bandas de absorção relacionadas à presença de água (Irons et al., 1989) puderam ser vistas em todas as curvas, independentemente do ângulo de visada nas proximidades de $1,40 \mu \mathrm{m}$ e, de forma mais acentuada, no comprimento de onda de $1,90 \mu \mathrm{m}$. A curva espectral apresentada pela série de solo McAllister apresentou a forma típica das descritas por Stoner \& Baumgardner (1981), indicativas de solos afetados pelos altos teores de Fe. Assim, as bandas de absorção mais intensas na região do azul e do infravermelho próximo (precisamente em 0,9 $\mu \mathrm{m}$ ) foram indicativas dessas características naquela série de solo. Outra banda de absorção presente nas curvas espectrais nas proximidades de $2,2 \mu \mathrm{m}$ se deve à presença de hidroxilas associadas a minerais silicatados (Irons et al., 1989). 
O comportamento anisotrópico desses três solos foi também estudado em relação à resposta espectral obtida no Nadir (FRB relativo) nas bandas do sensor TM localizadas no visível, infravermelho pró-
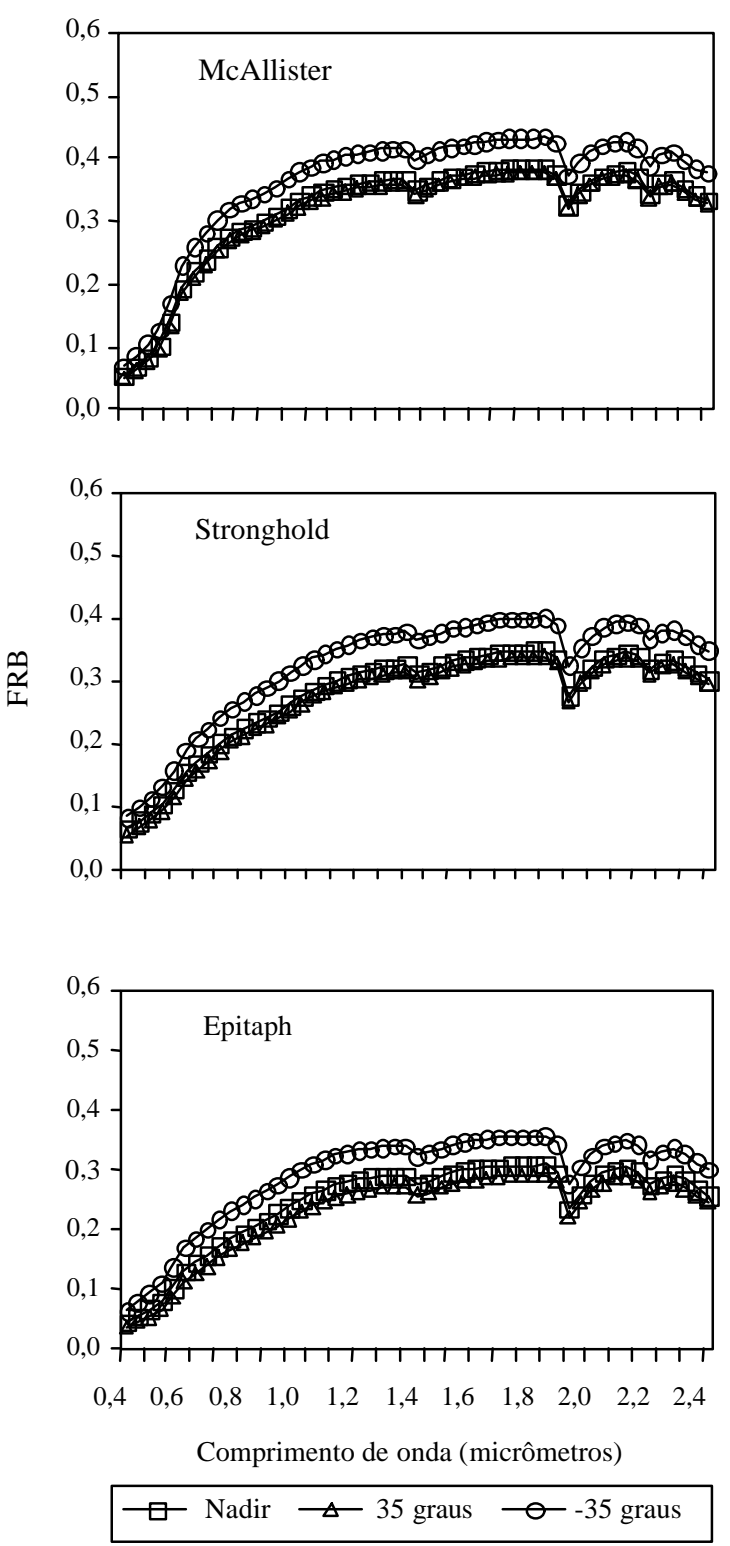

FIG. 3. Variação do fator de refletância bidirecional (FRB) obtido em laboratório (RELAB) em razão dos ângulos de visada do Nadir, -30 e 30 graus. ximo e infravermelho médio (Fig. 4). Assim como nos dados de campo, o anisotropismo das respostas espectrais obtidas pelo RELAB decresceu na direção do infravermelho médio; no entanto, em virtude das diferenças de escala e dos métodos de obtenção dos dados (Curran, 1994), esse anisotropismo foi
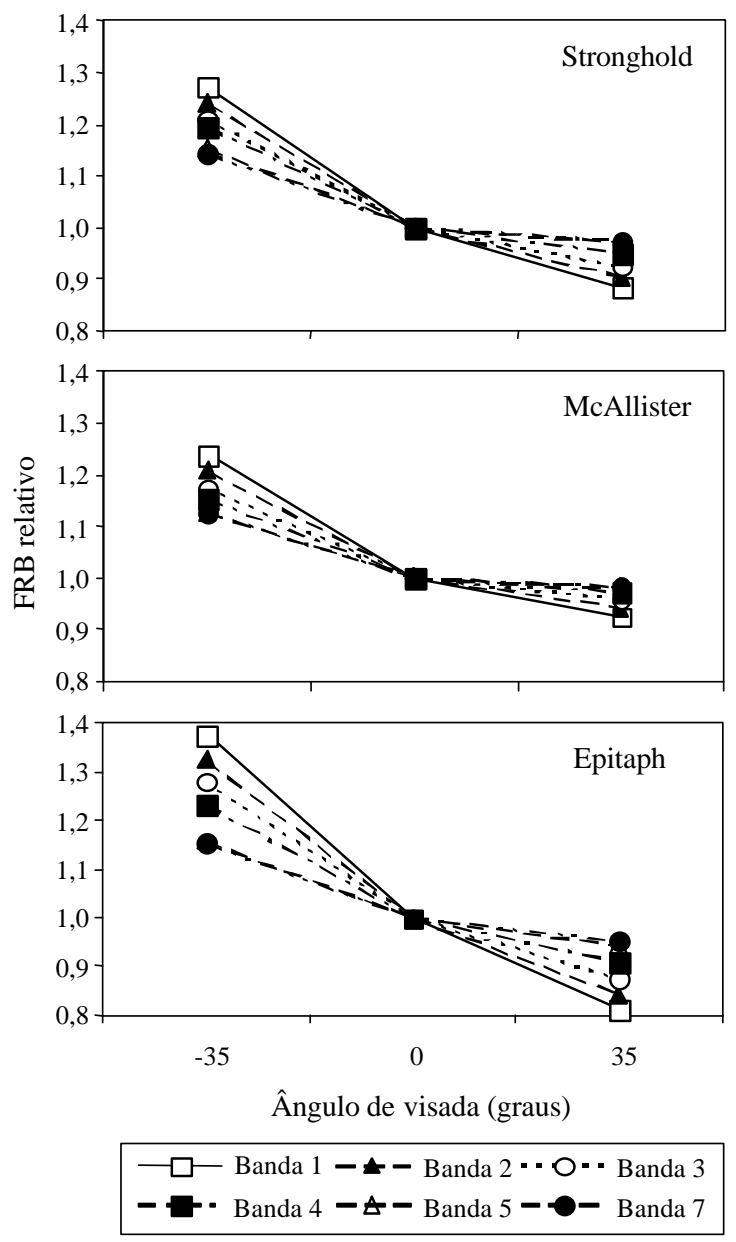

FIG. 4. Variação do fator de refletância bidirecional (FRB) obtido em laboratório (RELAB) em razão dos ângulos de visada do Nadir, -30 e 30 graus. (Os dados foram normalizados em relação ao Nadir; os símbolos indicam a resposta espectral das seis bandas do sensor TM do Landsat 5 localizadas no visível e nos infravermelhos próximo e médio, e simuladas a partir dos dados espectrorradiométricos). 
ressaltado nos dados do RELAB. Assim sendo, na banda azul (TM 1 ou 0,45 a $0,52 \mu \mathrm{m}$ ) e na direção do retroespalhamento $\left(-35^{\circ}\right)$, valores de 1,4 vez a resposta do Nadir, foram obtidos no solo Epitaph. Nas mesmas condições de ângulo de visada, esse valor caiu para, aproximadamente, 1,15 nas bandas $5(1,55$ a $1,75 \mu \mathrm{m})$ e 7 (2,08 a 2,35 $\mu \mathrm{m})$. Na região do espalhamento da radiação refletida, verificou-se uma menor expressão das propriedades anisotrópicas dos solos estudados, com valores de FRB relativo que variaram, na série de solo Epitaph, entre 0,8 (TM 1) e próximos a 1 (TM 5 e TM 7). Os comportamentos anisotrópicos apresentados pelas séries de solo McAllister e Stronghold foram semelhantes ao apresentado pela série de solo Epitaph, porém em menor intensidade (Fig. 4). Embora a microrrugosidade superficial das amostras desses solos não tenha sido avaliada, esse fator deve estar associado às diferenças no anisotropismo encontrado nas condições de laboratório relativas a esse três solos. Assim sendo, o solo Epitaph pode ser apontado como o que apresentou maior microrrugosidade superficial.

\section{Efeito da rugosidade sobre o FRB do solo Epitaph}

Os índices de rugosidade medidos no campo, nas superfícies lisa, intermediária e rugosa, são apresentados na Tabela 2. Verifica-se que, enquanto em dado ângulo solar-zenital esses tipos de superfície foram consistentemente diferentes, em dada condição de rugosidade esses índices mostraram independência em relação ao ângulo solar-zenital considerado. As superfícies intermediária e rugosa apresentaram, respectivamente, valores de comprimento de arco

TABELA 2. Índice de rugosidade de cada superfície ${ }^{1}$.

\begin{tabular}{cccc}
\hline $\begin{array}{c}\text { Ângulo } \\
\text { solar-zenital } \\
\text { (graus) }\end{array}$ & \multicolumn{3}{c}{$\begin{array}{c}\text { Rugosidade } \\
\text { (comprimento relativo de arco) }\end{array}$} \\
\cline { 2 - 4 } & Lisa & Intermediária & Rugosa \\
\hline 30 & $1,028 \mathrm{a}$ & $1,143 \mathrm{~b}$ & $1,304 \mathrm{c}$ \\
40 & $1,032 \mathrm{a}$ & $1,152 \mathrm{~b}$ & $1,293 \mathrm{c}$ \\
50 & $1,036 \mathrm{a}$ & $1,171 \mathrm{~b}$ & $1,341 \mathrm{c}$ \\
\hline
\end{tabular}

relativo em torno de $14 \%$ e $28 \%$ superiores aos apresentados pela superfície lisa.

A Tabela 3 apresenta as distribuições das frações de solo, pedregosidade e matéria orgânica presentes em cada tipo de superfície e medidas no plano principal de cada um dos ângulos solar-zenitais. Solo descoberto e cascalhos finos predominaram na superfície lisa, enquanto frações grosseiras maiores que cascalhos finos predominaram nas superfícies intermediária e rugosa. As diferenças entre as superfícies intermediária e rugosa foram muito mais devidas às proporções dos diferentes tipos de frações grosseiras do que ao total dessas frações. Desta forma, iguais proporções de cascalhos médios, cascalhos grandes e calhaus dominaram a superfície de rugosidade intermediária, enquanto a fração calhaus dominou a superfície rugosa.

O FRB relativo, nas diferentes condições de rugosidade e de ângulo solar-zenital, é apresentado, na Fig. 5, nos dados reamostrados da banda 1 do sensor TM Landsat 5. A banda 1 do TM foi escolhida porque os dados de campo e do RELAB evidenciaram maior comportamento anisotrópico nesta banda, quando comparado às demais bandas daquele sensor localizadas no visível e no infravermelho (Figs. 1 e 3). O ângulo de visada em que a refletância foi máxima em comparação com os dados do Nadir situou-se na direção do retroespalhamento e coincidiu com o ângulo solar-zenital em cada caso considerado. Assim, em um ângulo solar-zenital de $30^{\circ} \mathrm{O}$ FRB relativo atingiu o valor máximo em um ângulo de visada de, aproximadamente, $-30^{\circ}$. Em virtude das limitações na faixa de ângulos de visada coberta pelo aparelho de FRB $\left(-45^{\circ}\right.$ a $\left.45^{\circ}\right)$, o valor máximo do FRB relativo não é evidenciado no ângulo solarzenital de $50^{\circ}$. Independentemente do tipo de rugosidade superficial, o anisotropismo aumentou com o aumento do ângulo solar-zenital. Desta forma, na região do retroespalhamento o FRB relativo máximo variou de 1,5 a 1,9 para $30^{\circ}$ e $50^{\circ}$ de ângulo solar-zenital, respectivamente (Fig. 5). As curvas apresentadas mostram que, mesmo na superfície lisa, o comportamento anisotrópico da faixa do azul foi relativamente elevado. Esse resultado foi atribuído principalmente à fração de cascalhos finos, cujos teores, por causa da distribuição espacial, aumentaram com o aumento do ângulo solar-zenital. O espaça- 
mento de $10 \mathrm{~mm}$ entre os pinos do medidor de rugosidade não foi sensível ao intervalo de diâmetros dessa fração granulométrica. De fato, a medição da rugosidade superficial tem sido relatada como de extrema dificuldade, e vários aparelhos, tanto os de contato que usam pinos igualmente espaçados (Henry et al., 1980; Radke et al., 1981) quanto os de sensores remotos (Huang et al., 1988; Huang \& Bradford, 1990; Robichaud \& Molnau, 1990), têm sido sugeridos por diversos autores, sem que até o momento haja a recomendação de um método-padrão.

A distinção entre os três tipos de superfície na direção do retroespalhamento apresentou diferentes resultados conforme o ângulo solar-zenital considerado. Em um ângulo solar-zenital de $30^{\circ}$, a superfície rugosa apresentou maior anisotropismo que as demais, ao passo que nenhuma diferença foi notada entre os comportamentos anisotrópicos das superfícies lisa e intermediária. As medições de FRB na direção do retroespalhamento nos ângulos solar-zenitais de $40^{\circ}$ e $50^{\circ}$ não ofereceram oportunidade de distinção entre os três tipos de superfícies. Uma melhor distinção desses três tipos de superfícies foi observada quando se consideraram os valores de FRB relativo na direção do espalhamento da energia refletida (ângulos de visada positivos). Tais resultados foram evidenciados nos ângulos de visada maiores do que $35^{\circ}$. Nesses ângulos, a superfície rugosa mostrou maior anisotropismo do que as superfícies lisa e intermediária. A explicação para o fato de que as diferenças no comportamento anisotrópico das três superfícies estudadas tenham sido mais perceptíveis na direção do espalhamento da energia refletida pode estar associada à coloração mais escura da fração grosseira em comparação com a cor da fração granulométrica fina da superfície do solo (Fig. 6). Na direção do retroespalhamento, o efeito da diminuição da sombra, que é mínima onde ocorre o pico de refletância, é diminuído pela contribuição das frações grosseiras à resposta espectral de cada superfície. $\mathrm{Na}$ direção do espalhamento, o incremento da sombra com o aumento do ângulo de visada provocado pela fração grosseira acentuou as diferenças no anisotropismo das superfícies lisa, intermediária e rugosa.

Os resultados apresentados neste trabalho devem ser considerados por ocasião da análise de dados de sensores remotos orbitais para a extração de informações sobre solos (como, por exemplo, no uso de imagens como material básico para o mapeamento de solos), procurando-se distinguir entre as diferenças espectrais intrínsecas aos solos considerados e as diferenças induzidas pela geometria, dadas pela combinação dos ângulos de visada e ângulos solares. Além disso, as informações do comportamento espectral, levando-se em conta o umedecimento e os aspectos de rugosidade (maior ou menor presença de frações grosseiras) e suas interações com o comprimento de onda, podem ser de grande utilidade na análise de informações de sensores remotos orbitais voltadas para o mapeamento de solos.

TABELA 3. Caracterização da cobertura em cada tipo de rugosidade superficial e em cada ângulo solar-zenital.

\begin{tabular}{|c|c|c|c|c|c|c|c|c|c|}
\hline \multirow[t]{3}{*}{ Tipo de cobertura } & \multicolumn{9}{|c|}{ Cobertura do solo (\%) } \\
\hline & \multicolumn{3}{|c|}{ Lisa } & \multicolumn{3}{|c|}{ Intermediária } & \multicolumn{3}{|c|}{ Rugosa } \\
\hline & $30^{\circ}$ & $40^{\circ}$ & $50^{\circ}$ & $30^{\circ}$ & $40^{\circ}$ & $50^{\circ}$ & $30^{\circ}$ & $40^{\circ}$ & $50^{\circ}$ \\
\hline Solo descoberto & 76,5 & 64,5 & 60,0 & 35,0 & 37,5 & 25,5 & 33,5 & 34,5 & 31,0 \\
\hline Frações grosseiras & 13,0 & 27,5 & 34,0 & 56,0 & 49,5 & 59,0 & 53,0 & 55,0 & 50,5 \\
\hline Cascalhos finos (2 - $5 \mathrm{~mm})$ & 12,5 & 27,5 & 32,5 & 7,5 & 8,5 & 11,5 & 0,5 & 0 & 0,5 \\
\hline Cascalhos médios (5 - 20 mm) & 0 & 0 & 0 & 18,5 & 16,0 & 15,5 & 2,0 & 1,0 & 0,5 \\
\hline Cascalhos grandes (20 - $75 \mathrm{~mm})$ & 0,5 & 0 & 0 & 16,5 & 13,5 & 15,0 & 4,0 & 6,0 & 9,0 \\
\hline Calhaus (75 - $250 \mathrm{~mm})$ & 0 & 0 & 1,5 & 13,5 & 11,5 & 17,0 & 26,0 & 45,5 & 38,0 \\
\hline Matacões $(250$ - $600 \mathrm{~mm})$ & 0 & 0 & 0 & 0 & 0 & 0 & 19,5 & 2,5 & 2,5 \\
\hline Resíduos orgânicos & 10,5 & 8,0 & 6,0 & 9,0 & 13,5 & 14,5 & 13,5 & 10,5 & 18,5 \\
\hline
\end{tabular}



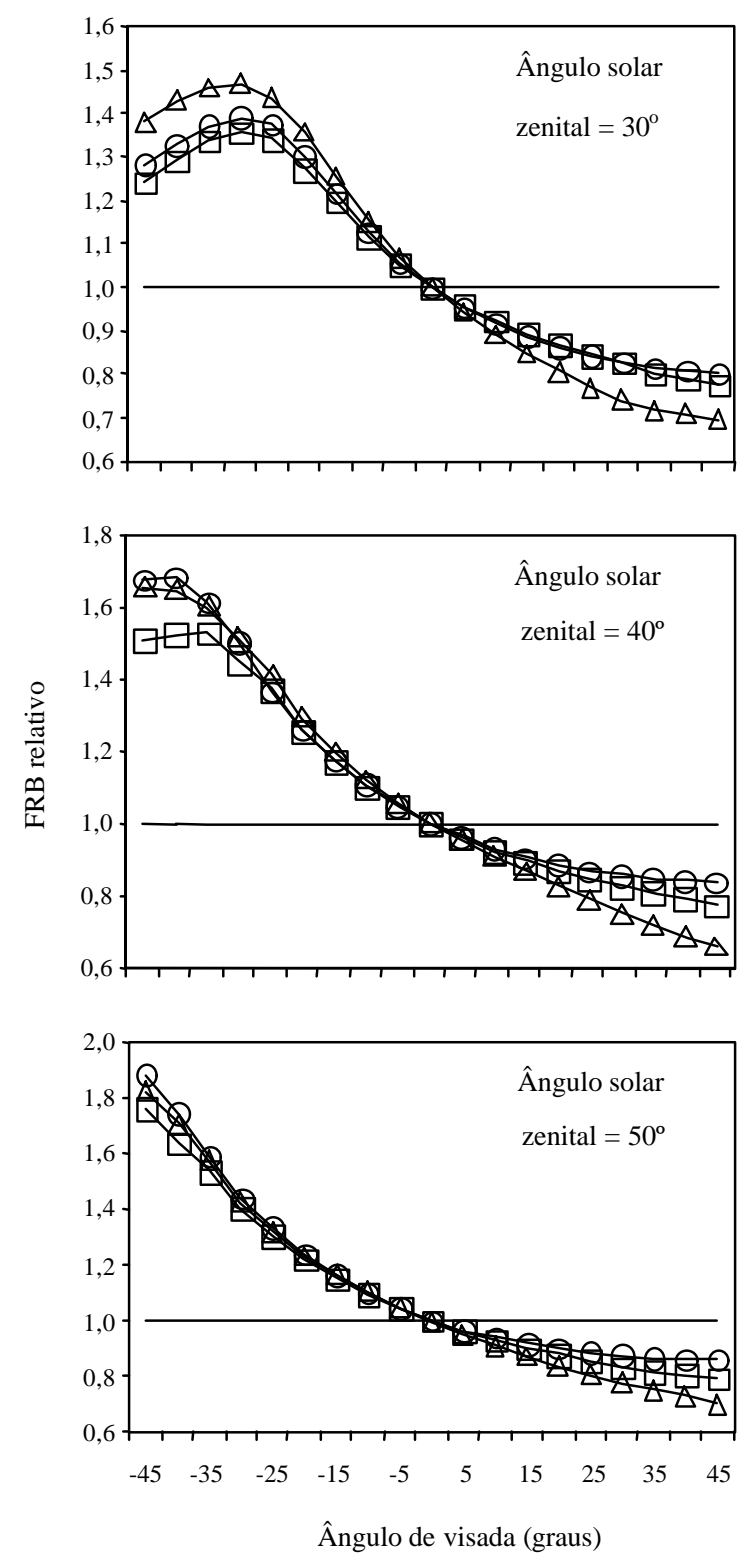

$\multimap$ Lisa $\neg \square$ Intermediária $\triangle$ Rugosa

FIG. 5. Variação do fator de refletância bidirecional (FRB) obtido no campo no solo Epitaph, em razão dos ângulos de visada e da rugosidade superficial. (Os dados foram normalizados em relação ao Nadir; os símbolos indicam a resposta espectral da banda 1 do sensor Landsat TM, simulada a partir dos dados espectrorradiométricos).

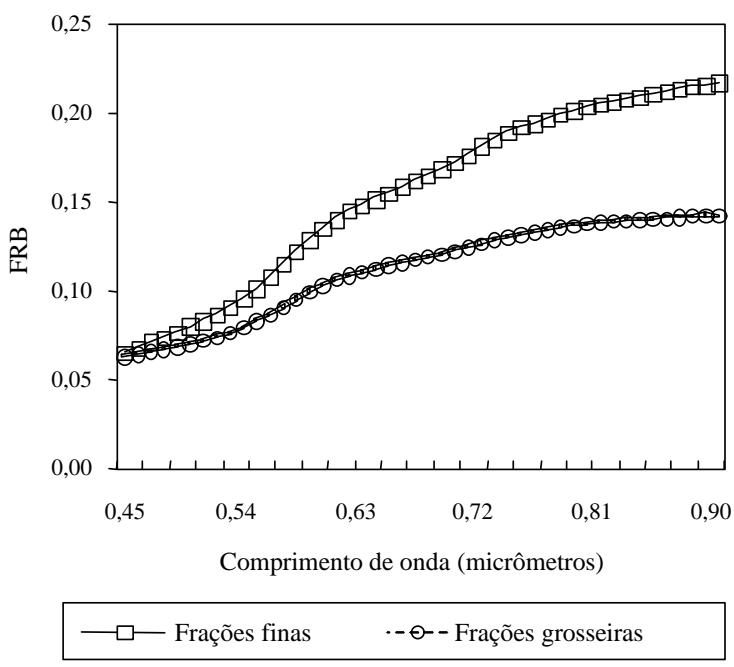

FIG. 6. Variação do fator de refletância bidirecional (FRB) obtido no campo para as frações finas e para as frações grosseiras (rochas) do solo Epitaph.

\section{CONCLUSÕES}

1. Os solos da microbacia experimental de Walnut Gulch (Arizona, EUA) apresentam comportamento anisotrópico assimétrico em relação à resposta obtida no ângulo de visada do Nadir.

2. O anisotropismo, além de ser diferente em relação a cada solo, mostra-se dependente da faixa do espectro considerada, do estado de umidade do solo, da geometria dada pelos ângulos de visada, e pelo ângulo solar-zenital, e da rugosidade superficial.

3. A série de solo Epitaph apresenta maior grau de anisotropismo do que as séries de solo McAllister e Stronghold.

4. O anisotropismo varia inversamente em relação às faixas do espectro consideradas, sendo maior no azul (banda 1 do sensor TM) do que nas bandas localizadas no infravermelho (bandas 4, $5 \mathrm{e}$ 7 do sensor TM).

5. As diferenças no comportamento anisotrópico dos solos são ressaltadas nas condições de laboratório.

6. O comportamento anisotrópico de todos os solos é reduzido quando suas superfícies são umedecidas. 
7. A resposta espectral em relação ao Nadir aumenta na direção do retroespalhamento.

8. O aumento da rugosidade superficial provoca elevação do anisotropismo da série de solo Epitaph.

\section{REFERÊNCIAS}

BOWERS, S.A.; HANKS, R.J. Reflection of radiant energy from soils. Soil Science, Baltimore, v.100, p.130-138, 1965.

COULSON, K.L. Effects of reflection properties of natural surfaces in aerial reconnaissance. Applied Optics, Washington, v.5, p.905-917, 1966.

COULSON, K.L.; BOURICIUS, G.M.; GRAY, E.L. Optical reflection properties of natural surfaces. Journal of Geophysical Research, Washington, v.70, p.4601-4611, 1965.

COULSON, K.L.; REYNOLDS, D.W. The spectral reflectance of natural surfaces. Journal of Applied Meteorology, Boston, v.10, p.1285-1295, 1971.

CURRAN, P.J. Imaging spectrometry: its present and future role in environmental research. In: HILL, J.; MEGIER, J. (Ed.). Imaging spectrometry: a tool for environmental observations. Dordrecht : Kluwer Academic, 1994. p.1-23.

CURRAN, P.J.; FOODY, G.M.; KONDRATYEV, K.Y.; KOZODEROV, V.V.; FEDCHENCKO, P.P. Remote sensing of soils and vegetation in the USSR. London : Taylor \& Francis, 1990. 265p.

EATON, F.D.; DIRMHIRN, I. Reflected irradiance indicatrices of natural surfaces and their effect on albedo. Applied Optics, Washington, v.18, p.995-1008, 1979.

EMBRAPA. Centro Nacional de Pesquisa de Solos (Rio de Janeiro, RJ). Manual de métodos de análise de solo. Rio de Janeiro, 1997. 212p. (Embrapa-CNPS. Documentos, 1).

EMBRAPA. Serviço Nacional de Levantamento e Conservação de Solos (Rio de Janeiro, RJ). Sistema brasileiro de classificação de solos: $2^{\underline{a}}$ aproximação. Rio de Janeiro, 1981. 107p.

EPIPHÂNIO, J.C.N.; FORMAGGIO, A.R.; VALERIANO, M.M.; OLIVEIRA, J.B. Comportamento espectral dos solos do Estado de São Paulo. São José dos Campos : Instituto Nacional de Pesquisas Espaciais, 1992. 132p. (INPE. Publicação, 5424-PRP/172).
ESTADOS UNIDOS. Department of Agriculture. Soil Survey Division. Soil Conservation Service. Soil Survey Staff. Soil survey manual. Washington, 1993. 437p. (USDA. Agriculture Handbook, 18).

ESTADOS UNIDOS. Department of Agriculture. Soil Survey Division. Soil Conservation Service. Soil Survey Staff. Soil taxonomy: a basic system of soil classification for making and interpreting soil surveys. Washington, 1975. 754p. (USDA. Agriculture Handbook, 436).

HENRY, J.E.; SCIARINI, M.J.; DOREN JUNIOR, D.M. van. A device for measuring soil surface profiles. Transactions of the ASAE, St. Joseph, v.23, p.1457-1459, 1980.

HOFFER, R.M.; JOHANNSEN, C.J. Ecological potentials in spectral signature analysis. In: JOHNSON, P.L. (Ed.). Remote sensing in ecology. Athens : University of Georgia Press, 1969. p.1-19.

HUANG, C.; BRADFORD, J.M. Portable laser scanner for measuring soil surface roughness. Soil Science Society of America. Journal, Madison, v.54, p.1402-1406, 1990.

HUANG, C.; WHITE, I.; THWAITE, E.G.; BENDELI, A. A non-contact laser system for measuring soil surface topography. Soil Science Society of America. Journal, Madison, v.52, p.350-355, 1988.

IRONS, J.R.; SMITH, J.A. Soil surface roughness characterization from light scattering observations. In: INTERNATIONAL GOSCIENCE AND REMOTE SENSING SYMPOSIUM, 1990. College Park. Proceedings. Washington : Institute of Electrical and Electronics Engineers, 1990. v.1, p.1007-1010.

IRONS, J.R.; WEISMILLER, R.A.; PETERSEN, G.W. Soil reflectance. In: ASRAR, G. (Ed.). Theory and applications of optical remote sensing. New York : J. Wiley, 1989. p.66-106.

JACKSON, R.D.; TEILLET, P.M.; SLATER, P.N.; FEDOSEJEVS, G.; JASINSKI, M.F.; AASE, J.K.; MORAN, M.S. Bi-directional measurements of surface reflectance for view angle corrections of oblique imagery. Remote Sensing of Environment, New York, v.32, p.189-202, 1990.

KIMES, D.S. Dynamics of directional reflectance factor distributions for vegetation canopies. Applied Optics, Washington, v.22, p.1264-1272, 1983. 
KIMES, D.S.; NEWCOMB, W.W.; TUCKER, C.J.; ZONNEVELD, I.S.; WIJNGAARDEN, W. van; LEEUW, J. de; EPEMA, G.F. Directional reflectance factor distributions for cover types of Northern Africa. Remote Sensing of Environment, New York, v.18, p.1-19, 1985.

KIMES, D.S.; SELLERS, P.J. Inferring hemispherical reflectance of the earth's surface for global energy budgets from remotely sensed nadir or directional radiance values. Remote Sensing of Environment, New York, v.18, p.205-223, 1985.

POST, D.F.; BRYANT, R.B.; BATCHILY, A.K.; HUETE, A.R.; LEVINE, S.J.; MAYS, M.D.; ESCADAFAL, R. Correlations between field and laboratory measurements of soil color. In: SOIL SCIENCE SOCIETY OF AMERICA (Madison, Estados Unidos). Soil color. Madison, 1993. p.35-49. (SSSA Special Publication, 31).

RADKE, J.K.; OTTERBY, M.A.; YOUNG, R.A.; ONSTAD, C.A. A microprocessor automated rillmeter. Transactions of the ASAE, St. Joseph, v.24, p.401-404, 1981.

REFLECTANCE EXPERIMENT LABORATORY (Providence, Estados Unidos). Reflectance Experiment Laboratory (RELAB): description and user's manual. Providence : Brown UniversityDepartment of Geological Sciences, 1993. 25p.

ROBICHAUD, P.R.; MOLNAU, M. Measuring soil roughness changes with an ultrasonic profiler. Transactions of the ASAE, St. Joseph, v.33, p.1851-1858, 1990.

SIMANTON, J.R.; DIXON, R.M.; McGOWAN, I. A microroughness meter for evaluating rainwater infiltration. In: HYDROLOGY AND WATER RESOURCES IN ARIZONA AND THE SOUTHWEST, 8., 1978, Flagstaff. Proceedings. Flagstaff : University of Arizona, 1971. p.171-174.

STONER, E.R.; BAUMGARDNER, M.F. Characteristic variations in reflectance of surface soils. Soil Science Society of America. Journal, Madison, v.45, p.1161-1165, 1981. 